\title{
A standardised storage solution for venepuncture/cannulation equipment could save an NHS hospital the equivalent of a whole junior doctor
}

\author{
Steven Lindley, lan Robertson
}

Royal United Hospital Bath NHS Trust

\begin{abstract}
Junior doctors, nursing staff, and phlebotomists spend a large proportion of their time taking blood samples and siting (venous) cannulae. Approximately 350 blood samples are taken daily across 25 wards at the Royal United Hospital Bath NHS Trust. There is no standard storage solution for venepuncture or cannulation equipment. On-call junior doctors cover most of the hospital's wards. Time is wasted locating essential equipment on unfamiliar wards and nurses are frequently interrupted to assist. These delays can compromise patient safety in emergencies as well as contributing to a source of daily inefficiency.
\end{abstract}

Junior doctors were timed collecting equipment needed for venepuncture and cannulation on unfamiliar wards. Initial results suggested large variation between timings on different wards. The medical admissions unit (MAU), which organises items for venepuncture and cannulation on a single trolley, was 4 times quicker than the mean of all other wards. MAU mean time $21.0 \mathrm{~s}$ vs. Non-standardised wards mean time $103.0 \mathrm{~s}$ $(p<0.0001)$. Estimates suggest approximately 47 hours per week (the equivalent of a fulltime doctor) could be saved by implementing a standard trust-wide storage solution.

We set out to introduce the MAU trolley format to all adult inpatient wards. All ward managers agreed to implement the trolley. 18 wards ( $72 \%$ of adult inpatient wards) already possessed the 'MAU style' trolley, which we standardised using an easy-to-follow inventory and laminated draw inlays. Feedback was very positive from doctors and ward staff alike. We repeated timings to validate the change and successfully presented a business case to senior management for a further 10 trolleys (£3623.78) for full adult inpatient ward coverage.

As junior doctors, we identified a common problem, tested solutions, and made early simple affordable changes. Initial work helped us present a compelling case for patient safety and efficiency improvements, releasing money to implement modest trust-wide quality improvement changes.

\section{Problem}

Junior doctors, nurses, and phlebotomists spend a significant proportion of their time taking blood samples and siting (venous) cannulae. Rotating jobs frequently and often covering unfamiliar wards out of hours, junior doctors find it difficult and time consuming to locate equipment from storage areas.

The Royal United Hospital (RUH) Bath NHS Trust has no standardised storage solution for venepuncture, arterial puncture, or cannulation equipment. This leads to time wasted locating essential equipment, interruptions to nursing staff, and selection of inappropriate equipment.

\section{Background}

An initiative known as 'The Productive Ward' focuses on improving ward processes and environments to enable nursing staff to spend more time on patient care and at the same time improve levels of safety and efficiency (1). This initiative has encouraged staff to organise equipment storage in a way that streamlines the delivery of patient care primarily to serve the nursing needs of a specific ward. Owing to the varying needs of different wards, this principle has lead to nonstandardised storage solutions across the Trust. Furthermore, equipment for venepuncture and cannulation are often disseminated across a storage area; for example gauze (for dressings) and syringes (for IV medication administration) are often grouped aiding nurse-related activities, separately.

Junior doctors spend much of their time taking blood samples and siting (venous) cannulae, often in emergency and time pressured situations. Different organisational approaches lead to time wasted locating equipment on unfamiliar wards. This leads to delays in urgent patient care and accumulative time lost which could be released to care. Disruptions to team working (frustration and interruption of nursing staff) and patient safety (staff 'cutting corners', using inappropriate equipment or staff using equipment from the crash trolley instead) are also potential consequences of disorganisation.

Junior doctors throughout the NHS repeatedly complain of wasting time finding equipment. Trainees in the London deanery estimate that each doctor wastes one hour locating equipment every day (2). Work by Sarkar and Ibitoye (2013) across 27 NHS Trusts, demonstrated that, foundation year one doctors were frustrated with poorly available and accessible equipment. Only $2.8 \%$ and $7.4 \%$ of doctors reported equipment availability as meeting 'an ideal 
standard' for cannulation and phlebotomy respectively (3).

It has long been recommended that resuscitation trolleys should be standardised throughout an institution (4). There was a time when the organisation of resuscitation equipment was at the discretion of the ward manager; a practice that is now unthinkable. Similarly, shouldn't the storage of venepuncture and cannulation equipment be standardised across each hospital? Apart from World Health Organisation guidelines suggesting that a tray or trolley should be used to take venepuncture equipment to the bedside of inpatients (5), formal standards do not stipulate standardised storage of such equipment.

Several standardised storage solutions have been reported at NHS Trusts to resolve these issues. Different storage solutions, implemented to good effect, reflect ward layout and funds available. Work at North Bristol NHS Trust resulted in the implementation of a standardised wall mounted bracket-box system, where as Blackpool Teaching Hospitals NHS Trust introduced a standardised trolley (anecdotes unpublished).

\section{Baseline Measurement}

We explored the scale of the problem with qualitative and quantitative evidence.

Junior doctor survey

An online survey was sent to 32 foundation year 1 (F1) doctors at the RUH. All F1 doctors were identified as those most likely to be involved in venepuncture/cannulation. Of the 21 respondents, $95 \%$ of doctors reported finding it either 'difficult' or 'very difficult' to locate all items needed for venepuncture and/or cannulation. As per figure $1,86 \%$ reported delays in emergency situations due to poor availability of equipment. Everyone expressed frustration current storage of equipment and believed that a trust-wide standard storage solution would be a positive improvement.

Junior doctor timed equipment collection

F1 Junior doctors were selected at random by bleeping the surgical and medical bleep-holders at $5 \mathrm{pm}$, asking whether they could spare approximately 10 minutes being times collecting equipment. All F1 doctors obliged. 15 doctors were timed collecting items for venepuncture and cannulation on a sample of 24 inpatient wards $(n=94)(f i g u r e 2)$. A standard selection of items was used in accordance with trust venepuncture protocols.

The authors timed collections, starting the clock after the task (and list of equipment) was understood by repeating it back to the timer, in the utility area of the designated ward. The clock was stopped when all items were collected and declared correctly by the subject. The clock kept running if the candidate had to seek help from ward staff. This strict criterion demonstrates both the reality of locating equipment on unfamiliar wards and prevents possible observer bias and inter-observer variability.

Data collection was undertaken in the first few weeks of the last of three rotations of the academic year. All doctors had experience of covering every adult inpatient wards on call at this point. We did not time doctors on wards where they had previously worked regularly, limiting the possibility of sampling bias.

The results highlighted significantly faster times on the MAU (Medical Assessment Unit) (venepuncture mean 21.2 seconds, $\mathrm{n}=19$ ) compared with all other wards combined (venepuncture mean 103.4 seconds, $\mathrm{n}=75$ (unpaired t-test: mean difference 82.1, $95 \% \mathrm{Cl} 58.5-105.8$ seconds $p<0.0001)$. Cannulation times were similar (venepuncture mean 23 seconds, $n=9$ vs. cannulation mean 112 seconds, $n=28$ ) (unpaired t-test, mean difference 83.4 seconds, $95 \%$ Cl 60.1-106.7 seconds, $p<0.0001)$.

These results reflect the improved accessibility of equipment that resulted from the use of a universal, comprehensively stocked equipment trolley, available across the 3 areas of the medical admissions unit.

See supplementary file: ds2317.png - "Figure 1: Baseline measurement: Time taken to collect a predetermined selection of venepuncture items"

\section{Design}

Initial pilot data suggested a trolley-based solution was effective and proved to be effective on MAU. We went on to carry out feasibility analysis on the potential benefit of implementing a similar trust-wide storage solution.

Of the 28 wards, $72 \%$ had access to identical trolleys, some already using them for venepuncture equipment storage with varying degrees of organization and others in use for bed linen or dressing storage.

Nursing and phlebotomy staff were generally welcoming of the proposal. Nursing staff acknowledged the difficulties of doctors locating equipment on unfamiliar wards, but expressed some concerns that 'it was another thing to have to restock'. Phlebotomy staff highlighted the benefit of a portable solution that can be taken to a patient's bedside and allowing them to relinquish the 'phlebotomy toolbox', carried between wards.

The MAU drawer layout was maintained. Four organised drawers provide venepuncture/cannulation equipment, whilst a fifth drawer is available for additional non-standard items. Laminated 'footprints' illustrating the contents were placed in the base of each drawer. A laminated inventory and drawer layout overview were also attached. Ward managers identified an individual responsible for restocking the trolley on a regular basis.

Consultation will begin on the suitability of the MAU drawer layout for all inpatient wards, after 4 months of standard implementation. This will allow staff to generate considered feedback and to coordinate formal review.

\section{Strategy}


BMJ Quality Improvement Reports

A proposal for Trust-wide standardisation was presented to all surgical and medical ward managers. We achieved unanimous support to implement a standardised trolley. Three ward managers of wards without the trolley thought the venture was such a good idea, they immediately placed orders with Bristol Maid. A formal presentation was given at the surgical and medical ward sisters meeting to confirm support and achieve a mandate for improvement.

We were limited in standardising pre-existent trolleys due to a paucity of draw dividers. Attempts were unsuccessfully made to source dividers from within the hospital, to 'make-good' preexisting trolleys. A business case was successfully presented to senior management to source dividers (£243) to allow us to initially introduce the standardised trolley to $72 \%$ of adult inpatient wards.

Feedback forms were placed on newly standardised trolleys, encouraging all staff to leave comments. We received feedback from doctors, nurses, phlebotomy staff, infection control and pharmacists. Suggestions for improvement included using free space to include lubricating jelly that doctors claim "they can never find" and the removal of alcohol wipes that the infection control department thought would be used inappropriately for skin preparation in cannulation (chlorhexidine is preferred). These improvements were implemented.

\section{Results}

In order to verify the effect of the trolley on newly standardised wards, we retimed junior doctors collecting equipment (figure 2). 46 recordings were taken across all wards, including non and newly standardised wards. Mean times for collecting equipment for venepuncture on standardised wards was 32.3 seconds compared to a mean of 118.6 seconds on wards which remained nonstandardised (unpaired t-test, mean difference 86.3 seconds, $95 \% \mathrm{Cl}$ 58.8-113.9 seconds, $\mathrm{p}<0.0001)$. Furthermore, statistical tests revealed no statistical difference between timings with MAU and newly standardised trolleys $(p=0.276)$. The significant reduction in time to collect equipment demonstrated two things. 1) We were able to successfully standardise the storage of blood taking equipment throughout a hospital and 2) trolleys were being well stocked, as equipment was readily available. We did not repeat timing for the collection of cannulation equipment, as the availability of phlebotomy equipment verifies baseline data and supports the introduction of a standardised trolley.

A final business case, including post standardisation data was presented to senior management for the funding of 10 Bristol Maid 'Caretray' trolleys (£3623.78). Funding was achieved to provide every adult inpatient ward with a trolley, prior to the start of new foundation year doctors to the Trust.

See supplementary file: ds2318.png - "Figure 2: Post measurement: Time taken to collect a predetermined selection of venepuncture items"

\section{Lessons and Limitations}

\section{Standardisation and Resistance to Change}

The proposal to standardise the storage of venepuncture and cannulation equipment was met with some reluctance at first. Several wards had dedicated venepuncture and cannulation trolleys (laid out differently, with inconsistent stock). Ward managers of a few wards who were already using 'phlebotomy' trolleys, were difficult to convince of the importance of standardisation. Criticism to the ergonomics of the layout of the MAU trolley lead to some ward managers requesting that we change the draw layout as a compromise. As the baseline data highlighted, the MAU trolley facilitated the quickest equipment collection times, suggesting this system was working well, we were reluctant to change draw design before we achieved standardisation across the trust. Once this has been achieved, we intend to consult on the layout of the trolley, should this issue continue to surface.

\section{Sustainability}

The success of the project will be defined by its sustainability. The key to sustainability is how well the trolleys are stocked. Although we asked ward managers to allocate responsibility to this, we are concerned, that if it is perceived that equipment can be found in general clinical storage areas as before, trolleys will not get replenished. I have encouraged ward managers to discuss with staff the benefit of the comprehensive trolley. We believe stocking will be achieved when the effect of a trust-wide standardised trolley is realised.

The RUH runs a programme known as 'Searchlight', providing work experience to teenagers with learning difficulties. Some students are given the responsibility of keeping clinical areas stocked. On graduating from the scheme, some wards opt to offer employment to ex-students. We are working with the organisers of Searchlight to increase the number of students to more wards.

\section{Efficiency Calculations}

Extrapolating time-savings demonstrated for venepuncture, we calculated potential efficiency savings associated with implementing a standardised storage solution.

In the large 565-bedded district general hospital of the $\mathrm{RUH}$, a mean of 353 sets of blood samples are taken on adult inpatient wards (excluding the Emergency Department) per weekday. 176 sets are taken per day at the weekend. With a time saving of 82.1 seconds per venepuncture episode, 48 hours 14 minutes could be saved per week with the introduction of a standardised trolley. This saving equates approximately to one full time junior doctor. Time saved can be 'released to care', rather than this providing an argument for reducing the number of junior doctors. There are also time-savings associated with cannulation, which we have not calculated.

At a cost of $£ 362.38$ per trolley, even if a new trolley had to be purchased for every ward in a hospital the size of the RUH (24 adult inpatient wards, excluding Intensive Care), the setup cost would be $£ 8,697$. Compared to the saving of a full time junior doctor (2013/14 


\section{BMJ Quality Improvement Reports}

salary £ 31,690 including $40 \%$ banding), this investment would be realised within 14 weeks of introduction.

\section{Conclusion}

Standardisation of routine venepuncture and cannulation equipment across a hospital has significant time efficiency benefits which likely impact on team working and patient safety outcomes. The lack of standardisation in the context of staff covering unfamiliar wards and rotating jobs regularly wastes the equivalent of a full time doctor in a large district general hospital. Our step-wide approach of identifying this problem, proposing an evidence-based solution, and affordably implementing changes with demonstrable effect enabled us to unlock hospital funding to make a significant system improvement.

Success of the standardised trolley will be dependent on how well the trolleys are stocked. Anticipating this, we identified possible solutions and will be reassessing stock levels, in order to sustainably embed the improvement in Trust service provision.

Junior doctors are faced with system inefficiencies within the NHS every day. Frequent job rotations mean that doctors have to get used to unfamiliar environments. Short rotations often increase doctor's tolerance to system inefficiencies, knowing they will only "have to put up with the problem" for a few months. There are many simple quality improvements to be made across the NHS. The standardisation of venepuncture and cannulation equipment is just one example of how empowering junior doctors to make changes can have efficiency and patient safety benefits.

As authors, our aim is to take this generalisable piece of work to other trusts lacking standardised venepuncture/cannulation storage solutions and suggest change, without having to re-demonstrate benefit.

\section{References}

1. NHS Institute for Innovation and Improvement [Internet]. London: NHS Institute; c2006-13 [cited 2013 Sept 28]. Available from: http://www.institute.nhs.uk/international/general/the producti ve ward.html

2. Roueche A, Hewitt J. 'Wading through treacle': quality improvement lessons from the frontline. BMJ Quality and Safety. 2012;(21):179-83

3. Sarkar $P$, Isitoye $R$. Setting the basics right: delays in phlebotomy and intravenous cannulation: a survey of foundation year 1 doctors. Clinical Medicine. 2013;(13): 239-43

4. Resuscitation Council UK. Cardiopulmonary Resuscitation: Standards for Clinical Practice and Training. London: Resuscitation Council (UK) Publication. [Published 2004 Oct; Updated 2008 Jun; cited 2013 Sept 28] $15 p$

5. World Health Organisation. WHO guidelines on drawing blood: best practices in phlebotomy: WHO Press, Switzerland. [Published 2010; cited 2013 November 19] 13

\section{Declaration of interests}

Nothing to Declare

\section{Acknowledgements}

Mr. Robert Bethune, Dr. Carol Peden, Dr. Leslie Jordan, Mrs. Lisa Lewis.

Steven Lindley and lan Robertson are joint first authors. 